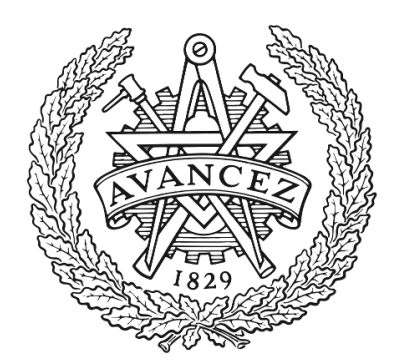

\title{
CHALMERS
}

UNIVERSITY OF TECHNOLOGY

\section{Optimization and Realization of Quadruple-ridge Flared Horn with New Spline-defined Profiles as a High-efficiency Feed over 4.6-24 GHz}

Downloaded from: https://research.chalmers.se, 2023-04-26 01:18 UTC

Citation for the original published paper (version of record):

Dong, B., Yang, J., Dahlström, J. et al (2019). Optimization and Realization of Quadruple-ridge Flared Horn with New Spline-defined Profiles as a High-efficiency Feed over 4.6-24 GHz. IEEE Transactions on Antennas and Propagation, 67(1): 585-590. http://dx.doi.org/10.1109/TAP.2018.2874673

N.B. When citing this work, cite the original published paper.

C2019 IEEE. Personal use of this material is permitted.

However, permission to reprint/republish this material for advertising or promotional purposes 


\section{Optimization and Realization of Quadruple-ridge Flared Horn with New Spline-defined Profiles as a High-efficiency Feed for Reflectors over $4.6-24 \mathrm{GHz}$}

Bin Dong, Jian Yang, Senior Member, IEEE, Jens Dahlström, Jonas Flygare, Miroslav Pantaleev, and Bhushan Billade, Member, IEEE,

\begin{abstract}
In this paper, we present a new optimization and realization of a quadruple-ridge flared horn (QRFH) as a feed for reflector antennas of the Square Kilometre Array (SKA) project. The QRFH has been numerically optimized by spline-defined profiles for both the ridges and the horn sidewall, with a conical cavity in the back-short for maximum aperture efficiency. The final aperture efficiency better than (for horizontal polarization) or around (for vertical polarization) $78 \%$ over $4.6-8 \mathrm{GHz}, \mathbf{7 0} \%$ over 8-15 GHz, 65\% over 15-20 GHz and 60\% over $20-24 \mathrm{GHz}$ in the SKA offset-Gregorian dual-reflector antenna has been achieved. The realization of the horn has been carried out carefully by applying several new mechanical design solutions in order to guarantee the accurate positioning of the ridges, the feeding pins and a good electrical contact. The measured $S_{11}$ is mostly better than $-10 \mathrm{~dB}$ and the predicted aperture efficiency based on the measured far field patterns agrees well with the simulated result. System performance, such as the sensitivity and system noise temperature, are also estimated and presented.
\end{abstract}

Index Terms - quadruple-ridge flared horn, wideband feed, spine-defined profiles, aperture efficiency, sensitivity.

\section{INTRODUCTION}

The Square Kilometre Array (SKA) [1] is an international project for the next-generation radio astronomical observations at meter- to centimeter-wavelength. It will be an interferometric array with a total collecting area of about one million square meters, which in its final stage will provide a sensitivity more than 50 times higher than the current largest interferometers in the world. The SKA will be constructed in two distinct phases, SKA1 and SKA2, the former being a subset of the latter. The SKA1 is now in its pre-construction phase. The Advanced Instrumentation Programme (AIP) is to enhance the SKA1 baseline and demonstrate an important technology prototype of direct relevance to SKA2 [2]. Sweden (Onsala

Manuscript received Sept. 19, 2017; revised March 2018. The design, manufacturing and testing of the Band-B feed was financed via the Planning Grant "Swedish contributions to the SKA radio-telescope in its pre-construction phase", Contract No C0546801. The project has also been partly supported by STINT project (CH2015-6360).

B. Dong is with the National Astronomical Observatories, Chinese Academy of Sciences and CAS Key Laboratory of FAST, NAOC, Chinese Academy of Sciences, 100012, Beijing, China, e-mail:dongbin@ nao.cas.cn. He visited Chalmers University of Technology/Onsala Space Observatory during 2016

- 2017, his visit and research were supported by the State Scholarship Fund from the China Scholarship Council (CSC No. 201504910308), the National Natural Science Foundation of China (11403053) and the Open Project Program of the Key Laboratory of FAST, NAOC, Chinese Academy of Sciences.

J. Yang is with the Dept. of Electrical Engineering, Chalmers University of Technology, S-41296 Gothenburg, Sweden. e-mail:jian.yang@chalmers.se

J. Dahlström, J. Flygare, M. Pantaleev, and B. Billade are with Onsala Space Observatory, Department of Space, Earth and Environment, Chalmers University of Technology, S-41296 Gothenburg, Sweden.

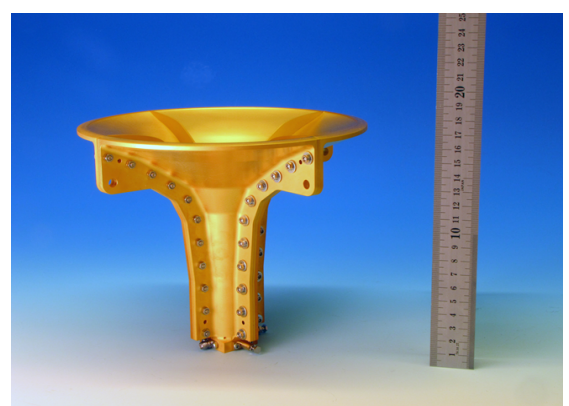

Fig. 1. Prototype of the spline-profiled quadruple-ridge flared horn for the SKA Band-B feed.

Space Observatory and Chalmers University of Technology) committed the technical research and design of the Band-B (4.6-24 GHz) feed for AIP.

Several ultra-wideband (UWB) feed technologies have been developed during the last decade. Among them the Eleven feed [3], [4] and the quadruple-ridge flared horn (QRFH) [5]-[7] are the most representative ones. The challenges in designing and realizing a Band-B feed include: 1) very challenging requirements for both aperture efficiency and sensitivity over a 5:1 bandwidth; 2) high frequency up to $24 \mathrm{GHz}$, therefore small dimensions and tight tolerances for manufacturing and assembling. In terms of the integration ability with LNA (Low Noise Amplifier), the feasibility for cryogenic operation and the flexibility of the beam shape to match with the SKA offset optics, a QRFH feed has advantages over an Eleven feed as the former offers standard single-ended $50-\Omega$ output ports, all metal structure and the freedom of controlling the beamwidth via changing its profiles.

The novelty of this work is the combination of existing and available ideas demonstrated previously in [5], [8], [9] to improve the QRFH feed. Contributions of this work include: 1) new spline-defined profiles for both the ridges and the horn sidewall of the QRFH, with the aim to achieve the very high aperture efficiency as well as sensitivity over a 5:1 bandwidth; 2) the realization of a $4.6-24 \mathrm{GHz}$ QRFH by implementing an edge-extrude mechanical technique to ensure good conductive contact between the ridges and the horn sidewall.

\section{DESIGN AND ANALYSIS}

The SKA dish antenna has a shaped offset-Gregorian optics, with a projected diameter of $15 \mathrm{~m}$ for the main reflector and $5.8 \mathrm{~m}$ for the sub-reflector with subtended angle of $\theta_{\mathrm{c}}=58^{\circ}$ and a shaped extension on the bottom side to reduce the ground spillover noise [10]. Specification for $\epsilon_{\text {ap }}$ and $A_{\text {eff }} / T_{\text {sys }}$ is listed in Table I. This requirement, if roughly estimated into the scenario of a primary-fed paraboloid, requires the feed to possess an $\epsilon_{\mathrm{ap}}$ of about $10 \%$ lower [7], [11], i.e. $68 \%$ over $4.6-8 \mathrm{GHz}, 60 \%$ over $8-15 \mathrm{GHz}, 55 \%$ over $15-20 \mathrm{GHz}$ and $50 \%$ over $20-24 \mathrm{GHz}$, which, to the best knowledge of the authors, have not been very well achieved by any existing 5:1 or 6:1 QRFHs [7], [12], [13].

The shapes of the horn sidewall and ridges are of great importance to the performance of QRFHs and have conventionally been expressed by analytical profiles [7]. However, 
TABLE I

THE SKA REQUIREMENT FOR $\epsilon_{\mathrm{ap}}$ AND $A_{\mathrm{eff}} / T_{\text {sys }}$ OVER BAND B

\begin{tabular}{lcccc}
\hline \hline frequency & $4.6-8 \mathrm{GHz}$ & $8-15 \mathrm{GHz}$ & $15-20 \mathrm{GHz}$ & $20-24 \mathrm{GHz}$ \\
\hline$\epsilon_{\mathrm{ap}}$ & $78 \%$ & $70 \%$ & $65 \%$ & $60 \%$ \\
\hline \multicolumn{5}{c}{} \\
\hline frequency & $4.6-13.8 \mathrm{GHz}$ & $13.8-20 \mathrm{GHz}$ & $20-24 \mathrm{GHz}$ \\
\hline$A_{\text {eff }} / T_{\text {sys }}$ & $6.1 \mathrm{~m}^{2} / \mathrm{K}$ & $4.7 \mathrm{~m}^{2} / \mathrm{K}$ & $3.5 \mathrm{~m}^{2} / \mathrm{K}$ \\
\hline \hline
\end{tabular}

QRFHs with such profiles suffer from a strong beamwidth variation over frequency (especially in H-plane) as well as the bad cross-polarization level (in D-plane, the maximum relative cross-polar level is only $-6 \mathrm{~dB}$ [7]), which makes the illumination efficiency $\epsilon_{\text {ill }}$ drop towards higher frequencies and results in a bad polarization efficiency $\epsilon_{\mathrm{pol}}$ and $\mathrm{BOR}_{1}$ efficiency $\epsilon_{\mathrm{BOR}_{1}}$. A lot of efforts were made to improve these sub-efficiencies, such as the utilization of quadraxial feeding [9], [12] and other kinds of aperture shapes [8], which make $\epsilon_{\mathrm{ill}}$ higher and more flat, but mostly at the cost of a worse spillover efficiency $\epsilon_{\mathrm{sp}}$ (barely up to $95 \%$ [7], [12]). In other words, $\epsilon_{\mathrm{ill}} \cdot \epsilon_{\mathrm{sp}}$ is still not good enough to meet the Band$\mathrm{B}$ requirement. The quadraxial feeding managed to improve the relative cross-polar level to $-9 \mathrm{~dB}$, but this differential technique needs additional baluns and hence more insertion loss would be introduced. Differential LNAs feeding can be another alternative, but it is normally more expensive and exhibits higher noise factors.

Spline-defined profile already showed very promising beam performance in [14] and is also adopted for the Band-B feed [15]. It is more flexible than the analytical profiles and enables a highly customizable optimization on the feed structure. After the optimization, the new spline-profiled Band-B feed has fulfilled and been very close to fulfill the SKA requirement for aperture efficiency for horizontal and vertical polarizations respectively, while still keeping the spillover noise temperature to an acceptable level.

\section{A. The Spline-defined Profiles of Band-B Feed}

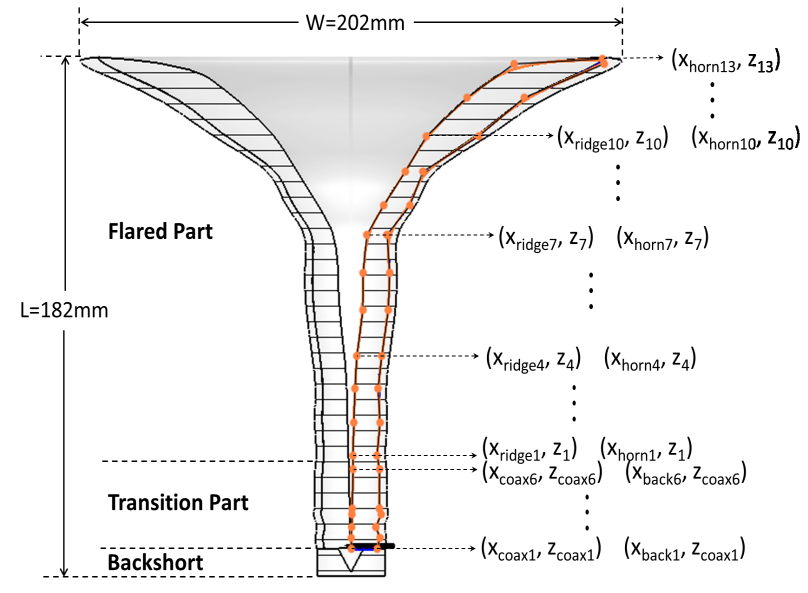

Fig. 2. Feed geometry: the spline-defined profiles defined by discrete points.

The proposed QRFH geometry is shown in Fig. 2, where the spline curve function in CST is utilized to construct the ridges and horn sidewall. In our design, 13 points ( $\mathrm{x}_{\text {horn1 }}$, $\left.\mathrm{z}_{1}\right),\left(\mathrm{x}_{\text {horn2 }}, \mathrm{z}_{2}\right),\left(\mathrm{x}_{\text {horn } 3}, \mathrm{z}_{3}\right), \ldots\left(\mathrm{x}_{\text {horn13 }}, \mathrm{z}_{13}\right)$ are chosen for the horn profile. 13 points with the same z-coordinates $\left(\mathrm{x}_{\text {ridge } 1}, \mathrm{z}_{1}\right),\left(\mathrm{x}_{\text {ridge } 2}, \mathrm{z}_{2}\right),\left(\mathrm{x}_{\text {ridge } 3}, \mathrm{z}_{3}\right), \ldots\left(\mathrm{x}_{\text {horn13 }}, \mathrm{z}_{13}\right)$ (the last point is shared with the horn profile) are chosen for the ridge profile. These 38 parameters in total form the flared part of the feed, as is shown in Fig. 2. The transition part is to have impedance transformation from the flared part to a standard $50-\Omega$ coaxial cable, where 6 points $\left(\mathrm{x}_{\mathrm{coax} 1}\right.$, $\left.\mathrm{z}_{\mathrm{coax} 1}\right),\left(\mathrm{x}_{\operatorname{coax} 2}, \mathrm{z}_{\mathrm{coax} 2}\right), \ldots\left(\mathrm{x}_{\mathrm{coax} 6}, \mathrm{z}_{\mathrm{coax} 6}\right)$ are defined for the inner profile of the ridges, and 6 points with the same $\mathrm{z}$-coordinates $\left(\mathrm{x}_{\text {back } 1}, \mathrm{z}_{\text {coax } 1}\right),\left(\mathrm{x}_{\text {back2 } 2}, \mathrm{z}_{\text {coax } 2}\right), \ldots\left(\mathrm{x}_{\text {back } 6}\right.$, $\mathrm{z}_{\mathrm{coax} 6}$ ) defined for the horn sidewall profile, 12 points as illustrated in Fig. 2. A conical-shaped backshort cavity, which possesses a good wideband performance, was utilized and defined by two parameters (radius and depth). The four ridges are made of metal pieces with thickness of $1.5 \mathrm{~mm}$, a large chamfering angle of $\theta=55.31^{\circ}$ is chosen to make the gap width between opposite ridges small enough. In total, there are 60 parameters in this spline-profiled QRFH feed.

The optimization procedure was divided into three stages: the flared part, the transition part and the whole feed. A time-efficient Matlab-CST optimization pipeline [7], [16] was established to search for the best performance with the Genetic Algorithm (GA). Please note that aperture efficiency $\epsilon_{\mathrm{ap}}$ was defined as the goal function and it was calculated in a primary-fed paraboloid using the method described in [17], [18]. Through intensive investigations, it was found that for optimizations in a primary-fed paraboloid, a smaller halfsubtended angle of $\theta_{\mathrm{c}}=51^{\circ}$ is more suitable than $\theta_{\mathrm{c}}=58^{\circ}$, as $\theta_{\mathrm{c}}=51^{\circ}$ provides a better control of the spillover efficiency $\epsilon_{\mathrm{sp}}$ and shows a better correlation with the corresponding aperture efficiency in the SKA dish. Over Band B, the $\epsilon_{\mathrm{ap}}$ in a primaryfed paraboloid with $\theta_{\mathrm{c}}=51^{\circ}$ would be constantly $\sim 10 \%$ lower than the final $\epsilon_{\text {ap__SKA }_{-}}$in the SKA dish, see Fig. 4.

Approximately 5000 different sets of parameters in total haven After optimization, the whole feed measures $202 \mathrm{~mm}$ in aperture diameter and $182 \mathrm{~mm}$ in length.

\section{B. Aperture Efficiency and Radiation Patterns}

The final optimized feed shows an aperture efficiency $\epsilon_{\mathrm{ap}}$ better than $68 \%$ over $4.6-8 \mathrm{GHz}, 60 \%$ over $8-15 \mathrm{GHz}, 58 \%$ over $15-20 \mathrm{GHz}$ and $55 \%$ over $20-24 \mathrm{GHz}$ in a primary-fed paraboloid $\left(\theta_{\mathrm{c}}=51^{\circ}\right)$, as shown in Fig. 4. The corresponding aperture efficiency in the SKA offset dish (the black-dashed curve) $\epsilon_{\text {ap_sKA, }}$, also shown in the figure, demonstrates a very similar trend with a constant $\sim 10 \%$ margin to $\epsilon_{\text {ap }}$. The illumination efficiency $\epsilon_{\mathrm{ill}}$ is higher than $65 \%$ over the entire bandwidth and exhibits a relatively flat trend, which implies nearly constant beamwidths over frequency. Meanwhile, the spillover efficiency $\epsilon_{\mathrm{sp}}$ is kept higher than $90 \%$ over 5-24 GHz and even exceeds 95\% towards mid-band and high-band, which provides a good management of the spillover noise temperature. The polarization efficiency $\epsilon_{\mathrm{pol}}$ is better than $95 \%$ over most of the band and falls down to slightly above $90 \%$ only around $11 \mathrm{GHz}$. This is a significant improvement compared to the analytical-profiled QRFHs' $\epsilon_{\text {pol }}$ of only about 

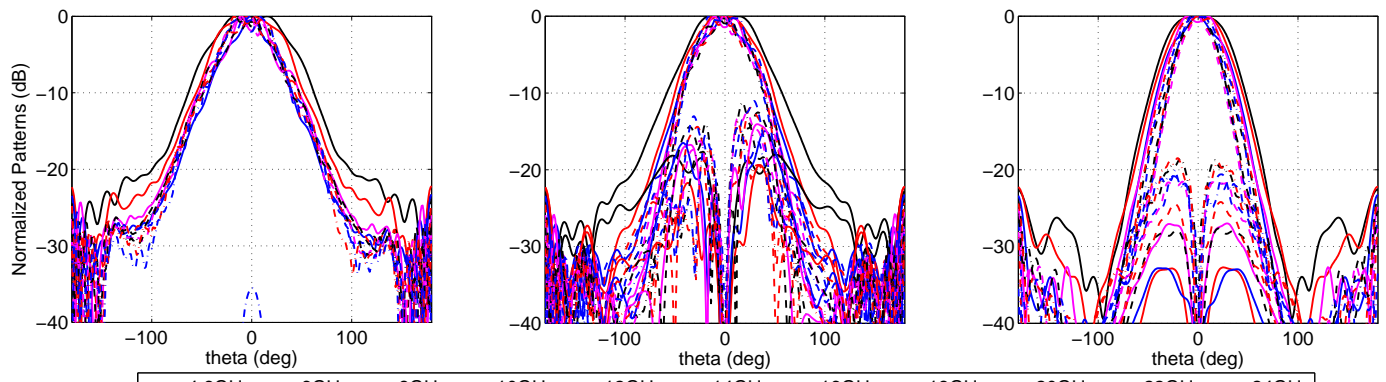

$4.6 \mathrm{GHz}-6 \mathrm{GHz}=8 \mathrm{GHz}-10 \mathrm{GHz}---12 \mathrm{GHz}---14 \mathrm{GHz}---16 \mathrm{GHz}---18 \mathrm{GHz} \cdot-\cdot 20 \mathrm{GHz} \cdot-\cdot 22 \mathrm{GHz} \cdot-\cdot 24 \mathrm{GHz}$

Fig. 3. Simulated radiation patterns in E-, D- and H-planes, both co-polar and cross-polar components are shown.

$90 \%$ across the bandwidth [13]. The $\mathrm{BOR}_{1}$ efficiency $\epsilon_{\mathrm{BOR}_{1}}$ is around $90 \%$ over the band, which is $5 \sim 10 \%$ higher than that of the QRFHs using analytical profiles [7].

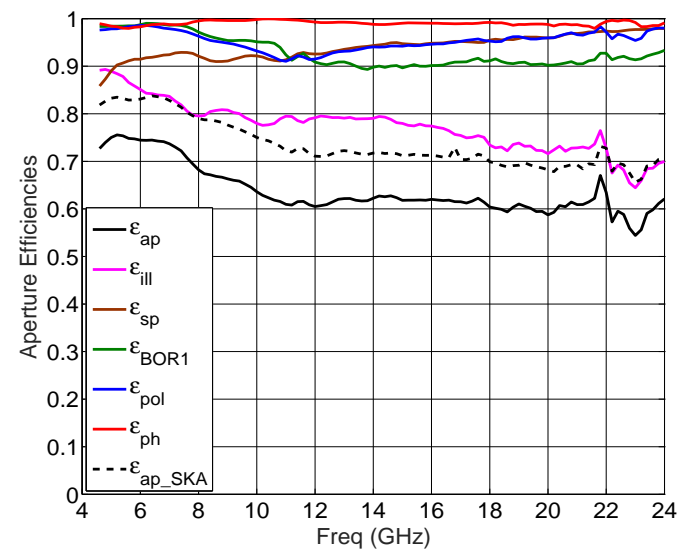

Fig. 4. Simulated aperture efficiency and the sub-efficiencies of the optimized spline-profiled QRFH feed, calculated in a primary-fed paraboloid with halfsubtended angle of $\theta_{\mathrm{C}}=51^{\circ}$.

Fig. 3 shows the simulated radiation patterns in $\mathrm{E}-\left(\phi=0^{\circ}\right)$, D- $\left(\phi=45^{\circ}\right)$ and $\mathrm{H}-\left(\phi=90^{\circ}\right)$ planes, normalized co-polar as well as relative cross-polar. The co-polar patterns exhibit very good Gaussian shapes with sidelobe and backlobe levels well below $-20 \mathrm{~dB}$. The beam profiles are nearly constant with frequency, even in $\mathrm{H}$-plane the fluctuation of nominal $10 \mathrm{~dB}$ beamwidth is only about $2 \times 20^{\circ}$, an improvement on the beam rotational symmetry from the existed QRFH's H-plane beamwidth fluctuation of $2 \times(30 \sim 35)^{\circ}$ [7], [13]. The crosspolar level is most significant in D-plane for BOR and quasiBOR antennas and the QRFH shows a relative cross-polar level below $-11 \mathrm{~dB}$ in D-plane, another improvement from the analytical-profiled QRFHs, which is about $-6 \mathrm{~dB}$ in [7], [13].

\section{Mechanical Realization}

The final electrical design was realized by dividing the whole feed into four pieces of ridges, four sidewall quadpieces, a backshort cavity and two feed pins, see Fig. 5(a) and Fig. 5(c), with each ridge being clamped between two adjacent sidewall quad-pieces, see Fig. 5(b). The ridges and sidewall quad-pieces were manufactured by Computer Numerical Controll (CNC) machines of Aluminum Alloy (AlSi1MgMn) which is cheap and also well-performed in vacuum and cryostat. On top of the metal surface, a gold layer of $3 \mu \mathrm{m}$ thick was plated to reduce thermal loss and prevent oxidation. All the small components (feed-pins, etc) were fabricated by using a watchmaker lathe.

Fig. 6 shows the simulated surface current on the inner sidewall of the horn at $24 \mathrm{GHz}$, where the transverse component of the current reaches its maximum at the jointing between the ridges and the sidewall, so any gaps between them will change the current distribution significantly, which will result in degraded reflection coefficient and radiation performance, and even leakage. A special edge-extrude technique is used to guarantee the good electrical contact: the sidewall quad-pieces were designed and manufactured with an edge extrusion with a height of $0.1 \mathrm{~mm}$ and a width of $0.2 \mathrm{~mm}$, see Fig. 5(e). Thus the sharp edge extrusion will indent the surface on both

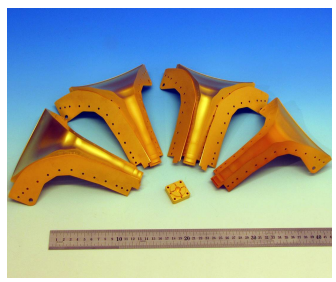

(a)

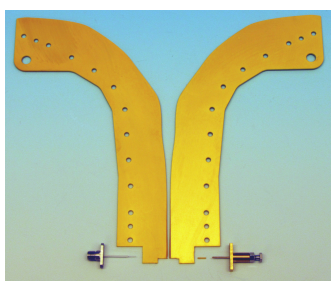

(c)

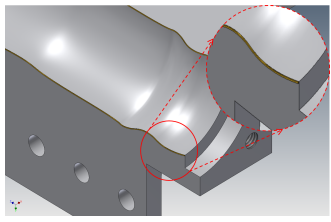

(e)

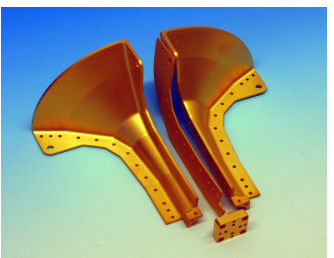

(b)

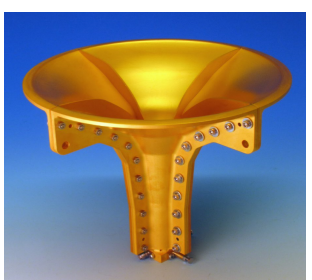

(d)

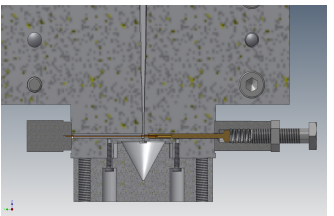

(f)
Fig. 5. Mechanical design of the Band-B feed: (a) manufactured major components; (b) zoom-in of the inner edge on one sidewall quad-piece (marked in yellow); (c) mechanical design around the feeding part; (d) each ridge is clamped between two quad-pieces during assembly; (e) assembly of the feeding pin; (f) the Band-B feed after assembly. 


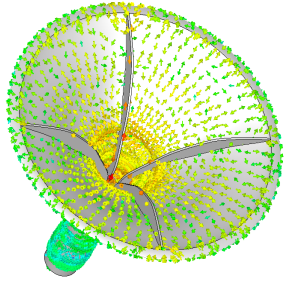

(a)

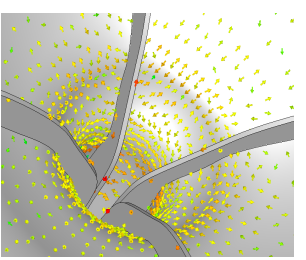

(b)
Fig. 6. Surface current distribution at $24 \mathrm{GHz}$ (in magnitude, based on CST simulation): (a) surface currents on the whole feed; (b) surface currents around the radiation region.

sides of each ridge as the ridge material is a bit softer and will act like a punching die. With the golden layer this would guarantee an extremely good electrical contact after tightening the two adjacent sidewall quad-pieces with screws.

A coaxial cable with an inner conductor diameter of $\phi=0.28 \mathrm{~mm}$ was chosen as the feeding pin. The inner conductor was inserted into the central hole of a spring-loaded cylinder stud embedded within the opposite ridge, which locks the pin by a chuck, see Fig. 5(f). This design secures the clamp of the pin and at the same time allows some small movements of the material due to heating- and cooling-extension.

\section{MeAsured PERFormanCE}

\section{A. Scattering Parameters}

The simulated and measured scattering parameters are shown in Fig. 7. The measured $S_{11}$ and $S_{22}$ are mostly below $-10 \mathrm{~dB}$ across the bandwidth except for around $10.6 \mathrm{GHz}$ and $23.2 \mathrm{GHz}$. At low frequencies, the measured results follow the same trend with simulation, while at high frequencies the measured reflection coefficient is higher than the simulation for about $5 \mathrm{~dB}$. The measured port isolation is around $-25 \mathrm{~dB}$ over the bandwidth. The inconsistency between measurement and simulation is caused mainly by a mechanical error, that on one ridge the feeding hole was not center-drilled due to a drift of the drill, the corresponding feed pin therefore has to be slightly bent in order to fit in.

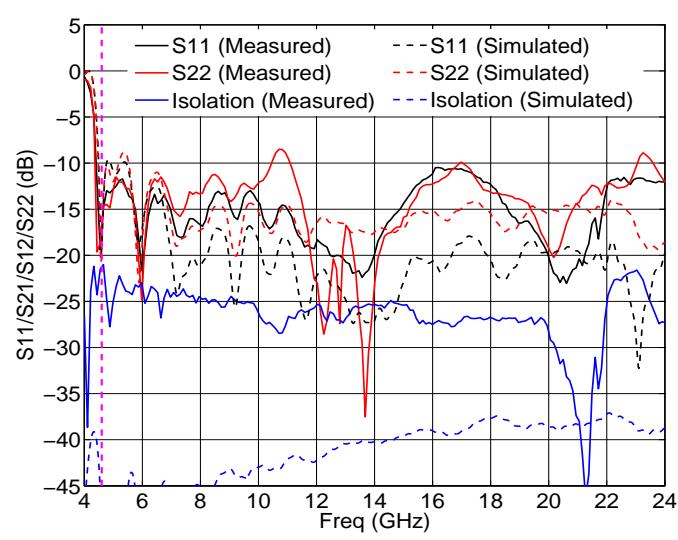

Fig. 7. Measured and simulated S-parameters of the prototype feed.

\section{B. Pattern Performance}

The feed radiation patterns were measured by the Antenna Measurement Team at Yebes Observatory in Spain. A spherical near-field measurement was conducted (Fig. 8) and five sets of Open Ended Waveguide (OEWG) probes (WR-229, WR159, WR-112, WR-75 and WR-42) were utilized to cover the entire Band B. The measurement frequency step is $0.2 \mathrm{GHz}$, but there was a gap between 15 and $15.6 \mathrm{GHz}$ that can't be covered by the aforementioned probes.

The measured radiation patterns show great consistence with simulations. Fig. 8 shows the measured and simulated $10 \mathrm{~dB}$ beamwidths in E-, D- and H-planes as well as the maximum relative cross-polar level (XPI) in D-plane. The measured relative cross-polar level in D-plane exhibits an expected performance of below $-11 \mathrm{~dB}$. Fig. 8 indicates the good beamwidth stability and therefore the rotational symmetry of radiation patterns has been improved.

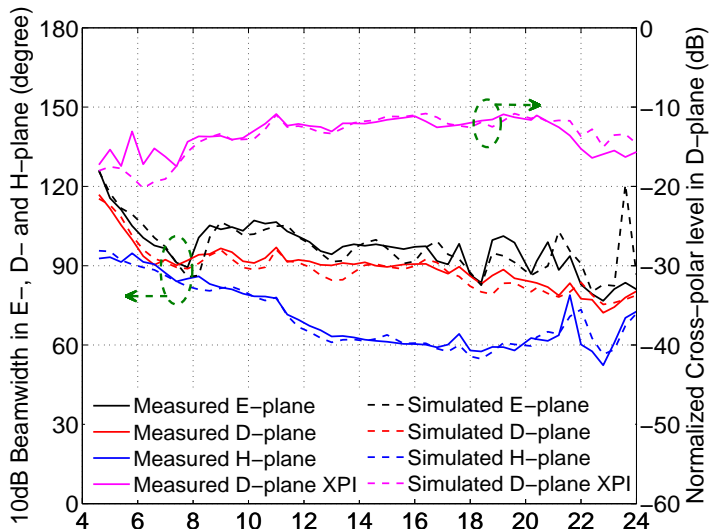

Fig. 8. Measured and simulated $10 \mathrm{~dB}$ beamwidth in E-, D- and H-planes, as well as the maximum relative cross-polar level (XPI) in D-plane.

\section{Predicted System Performance in the SKA Dish}

With the measured radiation patterns and estimated receiver noise, both aperture efficiency and sensitivity $\left(A_{\text {eff }} / T_{\text {sys }}\right)$ in the SKA shaped offset-Gregorian dual-reflector antenna (geometry shown in Fig. 9) were analyzed afterwards, using a System Simulator code written in Matlab [19], which models the total antenna beam pattern through physical optics and physical theory of diffraction calculations in GRASP. The effective area $A_{\text {eff }}$ is calculated from the theoretical aperture

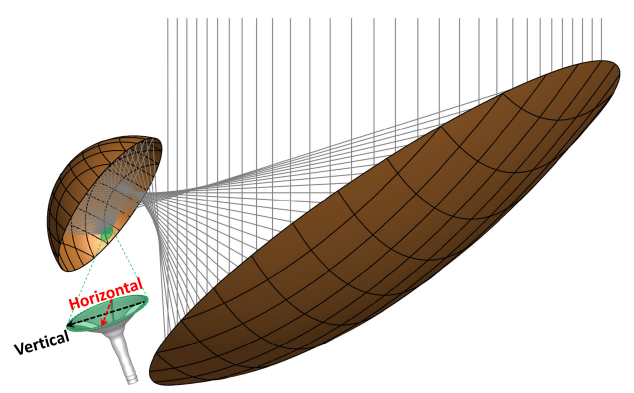

Fig. 9. The SKA dish geometry: an offset-Gregorian dual-reflector antenna. 
efficiency that is derived from the antenna directivity. A sky noise temperature model [20] for all elevations, specific to the conditions of the suggested SKA-mid site (altitude $1000 \mathrm{~m}$ ), is weighted with the antenna beam pattern and integrated over all elevations of the sky and the hot ground to calculate the total antenna noise $T_{\mathrm{A}}$. This sky noise temperature model mainly includes cosmic microwave background (CMB, $\sim 2.7 \mathrm{~K}$ ), the atmospheric noise and quite weak galactic emission component away from the galaxy plane. Please note that over Band $\mathrm{B}$ there is a significant increase in atmospheric noise around the water vapor absorption line at $22.2 \mathrm{GHz}$, as indicated by Fig. 2 and Fig. 3 in [20].

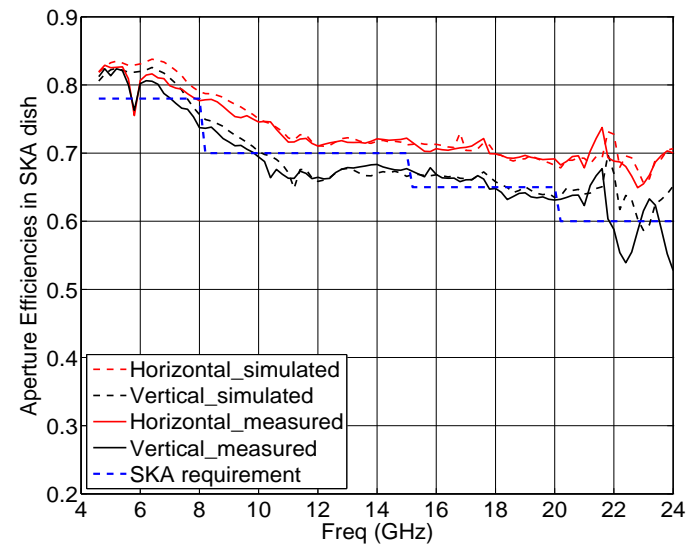

Fig. 10. Predicted aperture efficiencies $\epsilon_{\mathrm{ap}}$ in the SKA dish, based on simulated and measured radiation patterns, with a blue-dashed line representing the SKA requirement.

Fig. 10 shows the aperture efficiencies in the SKA dish, based on simulated patterns and measured patterns respectively, with the blue-dashed line representing the SKA requirement. The horizontal polarization has clearly fulfilled the requirement, with its $\epsilon_{\mathrm{ap}}$ better than $70 \%$ across most of the bandwidth, and the measurement agrees quite well with simulation except for the $5.8 \mathrm{GHz}$-dip and some minor reduction of $\sim 1 \%$ at lower band $(4.6-10 \mathrm{GHz})$. As for the vertical polarization, the measurement exhibits the same trend with simulation but drops to some extent over $20-24 \mathrm{GHz}$, this is partly due to the worse performance caused by the bent feeding pin in this polarization, as well as the polarization discrepancy that is caused by the offset optics of the SKA dish. Though not fully qualified, the vertical polarization is still close to the SKA requirement, and an aperture efficiency of better than $53 \%$ could be obtained over the entire bandwidth.

The estimated system noise temperature $T_{\mathrm{sys}}$ is shown in Fig. 11. It consists of the receiver noise temperature $T_{\mathrm{REC}}$ and the antenna noise temperature $T_{\mathrm{A}} . T_{\mathrm{REC}}$ includes the feed noise temperature $T_{\text {feed }}$ (in the cryostat) and LNA noise temperature $T_{\mathrm{LNA}}$ (the ohmic losses of coaxial cable and possible directional couplers for calibration between the feed and the receiver were not included). $2 \mathrm{~K}$ is estimated for $T_{\text {feed }}$ which covers the noise contributions from the feed window, the insertion loss of the feed and the mismatch loss between feed and 1st LNA. The LNA was designed by the Gothenburgbased company LNF (Low Noise Factory $\mathrm{AB}$ ) as part of the WBSPF development program and the measured $T_{\mathrm{LNA}}$ at ambient temperature of $20 \mathrm{~K}$ was used for estimation. The antenna noise temperature $T_{\mathrm{A}}$ is composed of the sky temperature $T_{\text {sky }}$ which is picked up by the main beam of the telescope, and the spillover noise temperature $T_{\mathrm{sp}}$ which is contributed from the hot ground and would be picked up through the sidelobes and backlobes of the telescope. Both $T_{\text {sky }}$ and $T_{\mathrm{sp}}$ were calculated by the System Simulator. In Fig. 11, the receiver noise $T_{\mathrm{REC}}$ is relatively flat and less than $10 \mathrm{~K}$ over the bandwidth, the antenna noise temperature $T_{\mathrm{A}}$ is comparable with $T_{\mathrm{REC}}$ at low-band and mid-band, but due to the strong water vapor absorption line around $22.2 \mathrm{GHz}$, $T_{\mathrm{A}}$ increases dramatically towards high-band, which is the main reason for the bad performance of $A_{\mathrm{eff}} / T_{\mathrm{sys}}$ at higher frequencies in Fig. 12.

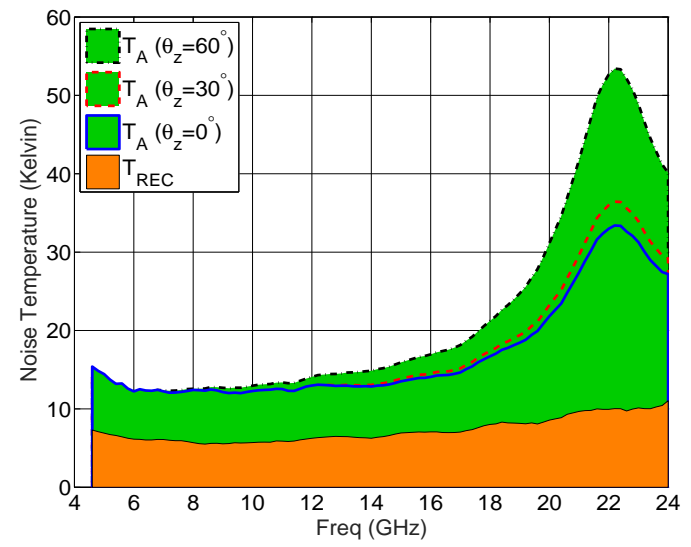

Fig. 11. Estimated system noise temperature over Band B, including the receiver noise $T_{\mathrm{REC}}$ and antenna noise $T_{\mathrm{A}}$.

The system sensitivities $\left(A_{\text {eff }} / T_{\text {sys }}\right)$ in the SKA dish for different zenith angles $\theta_{z}$, are given in Fig. 12. For $\theta_{z}=0^{\circ}$, the discrepancy between two polarizations is most severe, especially at the low-band, which is caused by the asymmetry of the radiation patterns and the offset optics of the SKA dish. However, as the zenith angle increases the discrepancy between two polarizations decreases. For $\theta_{\mathrm{z}}=0^{\circ}$ and $\theta_{\mathrm{z}}=30^{\circ}$, the sensitivity have fulfilled the SKA requirement over 4.6$21 \mathrm{GHz}$, but over $21-24 \mathrm{GHz}$ the vertical polarization fails to achieve the requirement. For $\theta_{z}=60^{\circ}$, both polarizations are performing well up until $18.25 \mathrm{GHz}$ but unqualified toward$\mathrm{s}$ higher frequencies, the worst performance occurs around $22.5 \mathrm{GHz}$ and it has a difference of about $1.5 \mathrm{~m}^{2} / K$ from the SKA requirement. The biggest problem at high frequencies here is caused by the water-vapor absorption line at $22.2 \mathrm{GHz}$, which is very severe in terms of sky-noise rather than spillover noise. This noise will be inevitably picked up through the main beam of the telescope. So, to achieve the sensitivity requirement at high frequencies, we would need a very high aperture efficiency but that would be very difficult to achieve for a wideband feed.

\section{CONClusion}

This paper presents the optimization and realization of a quadruple-ridge flared horn with new spline-defined profiles designed for the Band-B feed and the system performance 


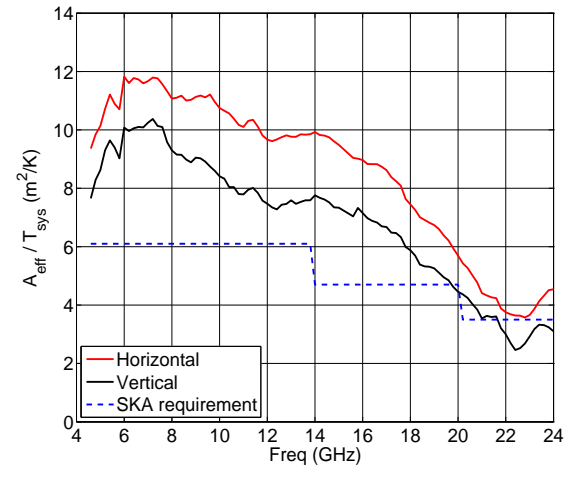

(a)

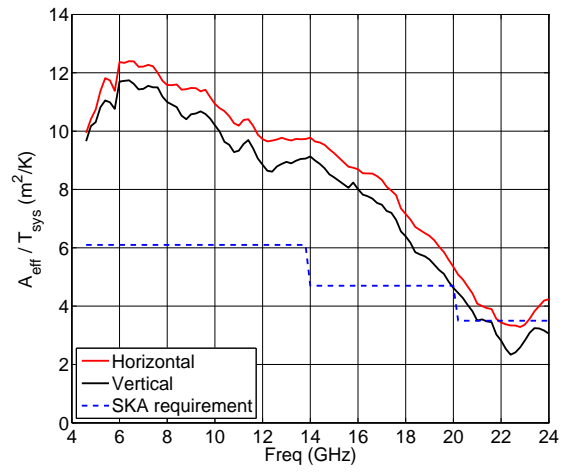

(b)

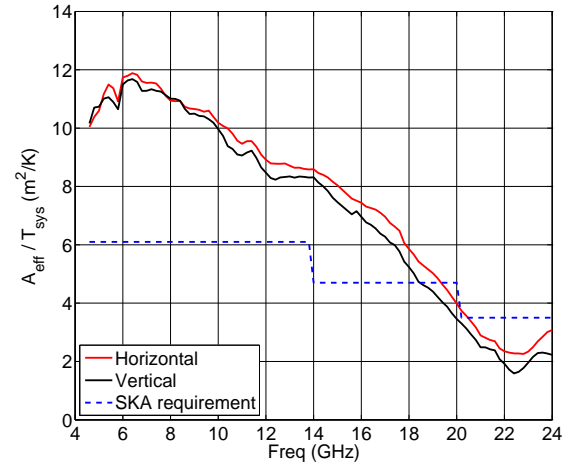

(c)

Fig. 12. Predicted system sensitivities of both polarizations for different zenith angle: (a) $\theta_{\mathrm{z}}=0^{\circ}$; (b) $\theta_{\mathrm{z}}=30^{\circ}$ and (c) $\theta_{\mathrm{z}}=60^{\circ}$.

analysis in the SKA dish. The feed exhibits a good impedance matching and beam performance over more than 5:1 bandwidth. Its sub-efficiencies and radiation patterns show that by adopting spline-defined profiles, more constant beamwidth and more symmetrical radiation patterns have been obtained, hence improved $\epsilon_{\mathrm{BOR}_{1}}, \epsilon_{\mathrm{pol}}$ and $\epsilon_{\mathrm{ill}} \cdot \epsilon_{\mathrm{sp}}$ can be achieved. An edge-extrude mechanical technique was implemented to realize a good contact between ridges and horn sidewall, so as to guarantee a high operational frequency. Measured results show reasonable agreement with simulations, predicted aperture efficiency in the SKA dish based on the measured farfield patterns have fulfilled the SKA requirement for its horizontal polarization, with a polarization discrepancy that makes the vertical polarization unqualified over part of the bandwidth. This discrepancy is caused by the asymmetrical optics of the SKA offset dish which are not fully opticalmatched with the symmetrically-designed Band-B feed, and thus was ignored during our primary-fed-parabolic optimization. As for the predicted $A_{\text {eff }} / T_{\text {sys }}$, a large margin above the requirement over low- and mid-band was achieved, while at high-band it slightly fails to satisfy the requirement due to the strong water-vapor absorption line around $22.2 \mathrm{GHz}$.

\section{ACKNOWLEDGMENT}

The authors would like to thank Heka Mechanical Engineering $\mathrm{AB}$ and Jocke at Micro-Slip HB for their valuable advices during the manufacture, and José Manuel Serna Puente, Dr. José Antonio López Fernández and Samuel López Ruiz from Yebes Observatory for their efforts on the pattern measurement.

\section{REFERENCES}

[1] R. Taylor, "The SKA: Recent developments," in 2015 Radio and Antenna Days of the Indian Ocean (RADIO), Sept 2015, pp. 1-2.

[2] M. Garrett, J. Cordes, D. Deboer, J. Jonas, S. Rawlings, and R. Schilizzi, "Square Kilometre Array: a concept design for Phase 1," arXiv preprint arXiv:1008.2871, 2010.

[3] J. Yang, M. Pantaleev, P.-S. Kildal, B. Klein, Y. Karandikar, L. Helldner, N. Wadefalk, and C. Beaudoin, "Cryogenic 2-13 GHz Eleven feed for reflector antennas in future wideband radio telescopes," IEEE Trans. Antennas Propag., vol. 59, no. 6, pp. 1918-1934, 2011.

[4] J. Yang, M. Pantaleev, B. Billade, M. Ivashina, T. Carozzi, L. Helldner, and M. Dahlgren, "A Compact Dual-Polarized 4-Port Eleven Feed With High Sensitivity for Reflectors Over 0.35-1.05 GHz," IEEE Trans. Antennas Propag., vol. 63, no. 12, pp. 5955-5960, Dec 2015.
[5] A. Akgiray, S. Weinreb, and W. Imbriale, "Design and measurements of dual-polarized wideband constant-beamwidth quadruple-ridged flared horn," in IEEE Int. Symp. Antennas Propag., July 2011, pp. 1135-1138.

[6] A. Akgiray and S. Weinreb, "Ultrawideband square and circular quadridge horns with near-constant beamwidth," in IEEE Int. Conf. UltraWideband. IEEE, 2012, pp. 518-522.

[7] A. Akgiray, S. Weinreb, W. A. Imbriale, and C. Beaudoin, "Circular quadruple-ridged flared horn achieving near-constant beamwidth over multioctave bandwidth: Design and measurements," IEEE Trans. Antennas Propag., vol. 61, no. 3, pp. 1099-1108, 2013.

[8] O. B. Jacobs, J. W. Odendaal, and J. Joubert, "Elliptically shaped quadridge horn antennas as feed for a reflector," IEEE Antennas Wireless Propag. Lett., vol. 10, pp. 756-759, 2011.

[9] T. S. Beukman, M. V. Ivashina, R. Maaskant, P. Meyer, and C. Bencivenni, "A quadraxial feed for ultra-wide bandwidth quadruple-ridged flared horn antennas," in 8th Eur. Conf. Antennas Propag.(EuCAP 2014), 2014, pp. 3312-3316.

[10] R. Lehmensiek, I. P. Theron, and D. I. L. de Villiers, "Deriving an optimum mapping function for the ska-shaped offset gregorian reflectors," IEEE Trans. Antennas Propag., vol. 63, no. 11, pp. 4658-4666, Nov 2015.

[11] D. I. L. de Villiers and R. Lehmensiek, "Dual reflector shaping for realistic frequency dependent feed patterns with specific secondary field pattern targets," in 8th Eur. Conf. Antennas Propag.(EuCAP 2014), April 2014, pp. 13-17.

[12] T. S. Beukman, P. Meyer, M. V. Ivashina, and R. Maaskant, "ModalBased Design of a Wideband Quadruple-Ridged Flared Horn Antenna," IEEE Trans. Antennas Propag., vol. 64, no. 5, pp. 1615-1626, May 2016.

[13] J. Shi, S. Weinreb, W. Zhong, X. Yin, and M. Yang, "Quadruple-ridged flared horn operating from 8 to $50 \mathrm{ghz}$," IEEE Transactions on Antennas and Propagation, vol. 65, no. 12, pp. 7322-7327, Dec 2017.

[14] B. Billade, J. Flygare, M. Dahlgren, M. Pantaleev et al., "A wideband feed system for SKA Band 1 covering frequencies from 350-1050 MHz," in 10th Eur. Conf. Antennas Propag.(EuCAP 2016). IEEE, 2016, pp. 1-3.

[15] J. Yang, J. Flygare, M. Pantaleev, and B. Billade, "Development of quadruple-ridge flared horn with spline-defined profile for band B of the Wide Band Single Pixel Feed (WBSPF) advanced instrumentation programme for SKA," in IEEE Int. Symp. Antennas Propag., June 2016, pp. $1345-1346$.

[16] A. Akgiray, "New Technologies Decade-Bandwidth Radio Astronomy: Quad-Ridged Flared Horn \& Compound-Semiconductor LNAs," Ph.D Thesis, California Institute of Technology, Pasadena, California, 2013.

[17] P. S. Kildal and Z. Sipus, "Classification of Rotationally Symmetric Antennas as Types BOR0 and BOR1," IEEE Antennas Propag. Mag., vol. 37, no. 6, pp. 114-117, Dec. 1995.

[18] P.-S. Kildal, Foundations of Antenna Engineering: A Unified Approach for Line-of-Sight and Multipath. Artech House, 2015.

[19] M. V. Ivashina, O. Iupikov, R. Maaskant, W. A. van Cappellen, and T. Oosterloo, "An Optimal Beamforming Strategy for Wide-Field Surveys With Phased-Array-Fed Reflector Antennas," IEEE Trans. Antennas Propag., vol. 59, no. 6, pp. 1864-1875, June 2011.

[20] G. Cortes-Medellin, "Antenna Noise Temperature Calculations," SKA Memo 95, July 2007. 